\title{
MARCAS DE DENTES DE CARNÍVOROS/CARNICEIROS EM MAMÍFEROS PLEISTOCÊNICOS DO NORDESTE DO BRASIL
}

\author{
HERMÍNIO ISMAEL DE ARAÚJO JÚNIOR \\ Programa de Pós-Graduação em Geologia, Departamento de Geologia, Instituto de Geociências, UFRJ, Av. Athos da \\ Silveira Ramos, 274, Ilha do Fundão, 21941-901, Rio de Janeiro, RJ, Brasil. herminio.ismael@yahoo.com.br \\ KLEBERSON DE OLIVEIRA PORPINO \\ Departamento de Ciências Biológicas, UERN, Av. Professor Antônio Campos, s/n, 59610-090, Mossoró, RN, Brasil. \\ kleporpino@yahoo.com.br \\ LÍLIAN PAGLARELLIBERGQVIST \\ Departamento de Geologia, Instituto de Geociências, UFRJ, Av. Athos da Silveira Ramos, 274, Ilha do Fundão, 21941-901, \\ Rio de Janeiro, RJ, Brasil. bergqvist@geologia.ufrj.br
}

\begin{abstract}
CARNIVORE/SCAVENGER TEETH MARKS ON PLEISTOCENE MAMMALS FROM THE NORTHEASTERN BRAZIL. In this work, the first occurrence of carnivore/scavenger teeth marks on Pleistocene fossil mammals collected in the Northeastern Brazil is reported. The fossils are from the "Tank of the Jirau", Itapipoca municipality, Ceará State, and are housed in the Museu de Pré-história de Itapipoca. The observed features, which are assigned to a largesized canid, correspond to scratches marks mostly perpendicular to the long axis of the bone, occurring mainly in ribs, vertebrae, tibiae and ulnae. Based on the size of the scratches, the geographical distribution and dietary habits of canids from the Late Pleistocene of Brazil, Protocyon troglodytes (Lund) is the most likely producer of the observed features, although this taxon is not represented in the Jirau fossil accumulation.
\end{abstract}

Key words: taphonomy, natural tanks, Pleistocene mammals, Protocyon troglodytes, Itapipoca, Northeastern Brazil.

\begin{abstract}
RESUMO - Neste trabalho é registrada pela primeira vez a ocorrência de marcas de dentes de carnívoros/carniceiros em fósseis de mamíferos pleistocênicos coletados no Nordeste do Brasil. Os fósseis são procedentes do Tanque do Jirau, em Itapipoca, Estado do Ceará, e se encontram depositados no Museu de Pré-história de Itapipoca. As feições observadas, atribuídas a um canídeo de grande porte, são do tipo "scracthes" e ocorrem principalmente em costelas, vértebras, tíbias e ulnas, sendo predominantemente perpendiculares em relação ao eixo maior dos ossos. Com base no tamanho das feições produzidas, na distribuição geográfica e nos hábitos alimentares dos canídeos do Pleistoceno tardio brasileiro, Protocyon troglodytes (Lund) é sugerido como o mais provável gerador das marcas, mesmo não estando representado na acumulação fossilífera do Jirau.
\end{abstract}

Palavras-chave: tafonomia, tanques naturais, mamíferos pleistocênicos, Protocyon troglodytes, Itapipoca, Nordeste do Brasil.

\section{INTRODUÇÃO}

Marcas de dentes de carnívoros/carniceiros, até o presente momento, não estão presentes no conjunto de assinaturas tafonômicas elencado para os depósitos de vertebrados pleistocênicos do Nordeste do Brasil (e.g. Araújo-Júnior \& Porpino, 2011). Por outro lado, essas feições foram registradas em fósseis de depósitos de mesma idade das regiões Norte e Sudeste do Brasil por Avilla et al. (2009) e Dominato et al. (2011).

A identificação e interpretação dessas feições são importantes para a inferência de aspectos ligados aos agentes de acumulação óssea (Haynes, 1980a; Andrews, 1990), aos vieses tafonômicos introduzidos pela atuação biológica na tanatocenose e à relação presa-predador (Palmqvist \& Arribas, 2001; Avilla et al., 2009). Além disso, essas feições podem servir como evidência da presença de carnívoros nas paleocomunidades quando estes não estão representados sob a forma de fósseis corporais.

Fósseis coletados durante a última década no Tanque do Jirau apresentam um complexo de feições tafonômicas, dentre as quais é possível reconhecer marcas de dentes de carnívoros/ carniceiros. O objetivo deste trabalho é descrever essas marcas e tentar associá-las a algum dos táxons de carnívoros conhecidos para as acumulações fossilíferas do Pleistoceno tardio brasileiro.

\section{MATERIAL E MÉTODOS}

Os fósseis foram coletados no Sítio Paleontológico do Jirau (Figura 1A), em um depósito do tipo tanque (Figura 2B), o qual está localizado $35 \mathrm{~km}$ a noroeste da sede do Município de Itapipoca, Estado do Ceará. O material estudado encontrase depositado na coleção paleontológica do Museu de Pré- 
história de Itapipoca (MUPHI), sob os números MUPHI 1576 a MUPHI 1601.

Os fósseis procedentes do Tanque do Jirau são atribuídos ao Pleistoceno tardio pela presença das espécies Notiomastodon platensis (Ameghino, 1888) [= Stegomastodon waringi (Holand, 1920); Mothé et al., no prelo] e Xenorhinotherium bahiense Cartelle \& Lessa, 1988, para as quais têm sido feitas datações absolutas com base em elementos esqueletais procedentes de outros tanques nordestinos (Kinoshita et al., 2005, 2008; Oliveira et al., 2009). Uma listagem mais detalhada dos táxons registrados até o momento na tafocenose do Jirau pode ser conferida em Ximenes et al. (2010).

Para identificação, as feições foram comparadas com aquelas representadas na literatura especializada (Haynes, 1980a, 1982, 1983; Lyman, 1994). Além disso, as peças em estudo foram comparadas com fotografias de espécimes portando feições semelhantes pertencentes à coleção paleontológica da University of Nevada (Universidade de Nevada), nos Estados Unidos da América.

\section{RESULTADOS E DISCUSSÃO}

Foram identificadas marcas de dentes de carnívoros/ carniceiros em 26 dos 4.149 elementos esqueletais coletados
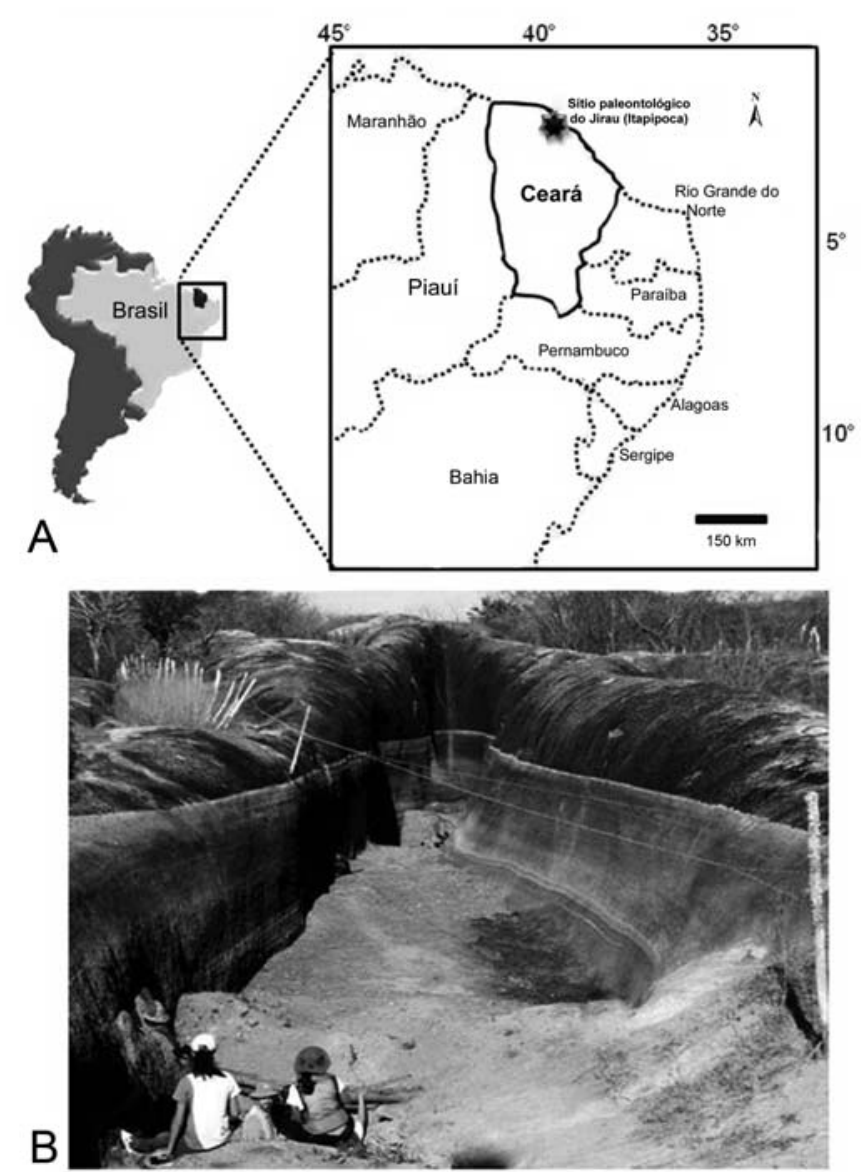

Figura 1. Mapa de localização do sítio paleontológico do Jirau (A) e visão geral do depósito fossilífero (B).

Figure 1. Location map of the Jirau paleontological site $(\mathbf{A})$ and general view of the fossiliferous deposit (B). no Tanque do Jirau. As marcas estão presentes em fósseis atribuídos às espécies Notiomastodon platensis, Eremotherium laurillardi Lund, 1842 e Glossotherium Owen, 1840 , todos táxons herbívoros e de grande porte. Os elementos esqueletais que apresentam as marcas de dentes são listados na Tabela 1. As ranhuras em ossos como fêmures, úmeros, tíbias (MUPHI 1601, Figura 2) e vértebras, de um modo geral, apresentam tamanho aproximado de $50 \mathrm{~mm}$ de comprimento por $3 \mathrm{~mm}$ de largura, enquanto que nas costelas, falanges, astrágalo e ulna variam de 25 a $30 \mathrm{~mm}$ de comprimento por $3 \mathrm{~mm}$ de largura (Tabela 1). Ocorrem principalmente nas faces medial e lateral do corpo do osso e estão orientadas perpendicularmente em relação ao maior eixo do osso (MUPHI 1591, Figura 3), podendo ser classificadas segundo Haynes (1980a) como do tipo "scratches".

Excluiu-se a possibilidade dessas feições terem sido produzidas durante a coleta ou preparação dos fósseis, pois neste caso a depressão resultante das ferramentas de preparação ou coleta apresentaria cor diferente da superfície óssea não afetada. Além disso, todos os elementos esqueletais apresentam marcas de dessecação possivelmente resultantes da exposição da tanatocenose ao intemperismo (sensu Behrensmeyer, 1978) e ranhuras provavelmente geradas por pisoteio antes do soterramento (sensu Britt et al., 2009), as quais se sobrepõem às feições aqui analisadas, o que mostra que estas não foram produzidas durante ou após a coleta.

De acordo com Haynes (1983), marcas de dentes do tipo "scratches" são geradas principalmente por canídeos de maior porte, os quais possuem o hábito de mordiscar ossos, arrastando seus dentes sobre a superfície e formando marcas lineares semelhantes a arranhões, usualmente perpendiculares em relação ao eixo maior do osso (ver Figura 4). Haynes (1983) assume que ossos com "scratches" posicionados dessa forma são característicos de carcaças em estágio final de utilização por carniceiros, quando resta uma pequena quantidade de tecidos moles recobrindo os ossos.

Felídeos e ursídeos, potenciais geradores de marcas de mordidas e outras alterações preservadas em fósseis (Haynes, 1980a, 1983), são registrados nos depósitos pleistocênicos brasileiros de acordo com Gomide et al. (1987), Trajano \& Ferrarezzi (1994), Cartelle (1998), Porpino et al. (2004) e Auler et al. (2006) (e.g. Panthera onca Linnaeus, 1758; Arctotherium brasiliense Lund, 1839; Smilodon populator Lund, 1842; Felis Linnaeus, 1758). No entanto, desconsiderase a inclusão dos táxons dessas famílias na lista dos possíveis geradores das feições aqui analisadas porque o padrão das marcas produzidas por esses animais é bastante distinto das marcas observadas. Ossos mordiscados por felídeos apresentam perfurações profundas localizadas principalmente nas porções proximais de ossos longos (e.g. fêmures), enquanto que os ursídeos deixam marcas de dentes associadas a fraturas paralelas ao maior eixo do osso ou semelhantes a marcas produzidas por roedores (Haynes, 1983). Padrões comparáveis não foram observados nos espécimes estudados.

Também foi excluída a hipótese das feições terem sido produzidas por pisoteio (trampling), pois em fósseis com 
esse tipo de marca os arranhões não apresentam um padrão de orientação definido (Fiorillo, 1984, 1991; Britt et al., 2009), diferentemente do que ocorre nos fósseis do Jirau, onde os elementos esqueletais possuem "scratches" paralelamente posicionados (ver Figuras 2-4).

Haveria a possibilidade das feições analisadas terem sido produzidas pelo homem, o qual pode deixar marcas de corte em ossos longos de mamíferos (que fazem parte de sua dieta) através da utilização de instrumentos líticos (Shipman, 1981; Lyman, 1994; Bissaro-Júnior, 2008). As marcas de corte que mais se assemelham às analisadas neste trabalho são as produzidas por

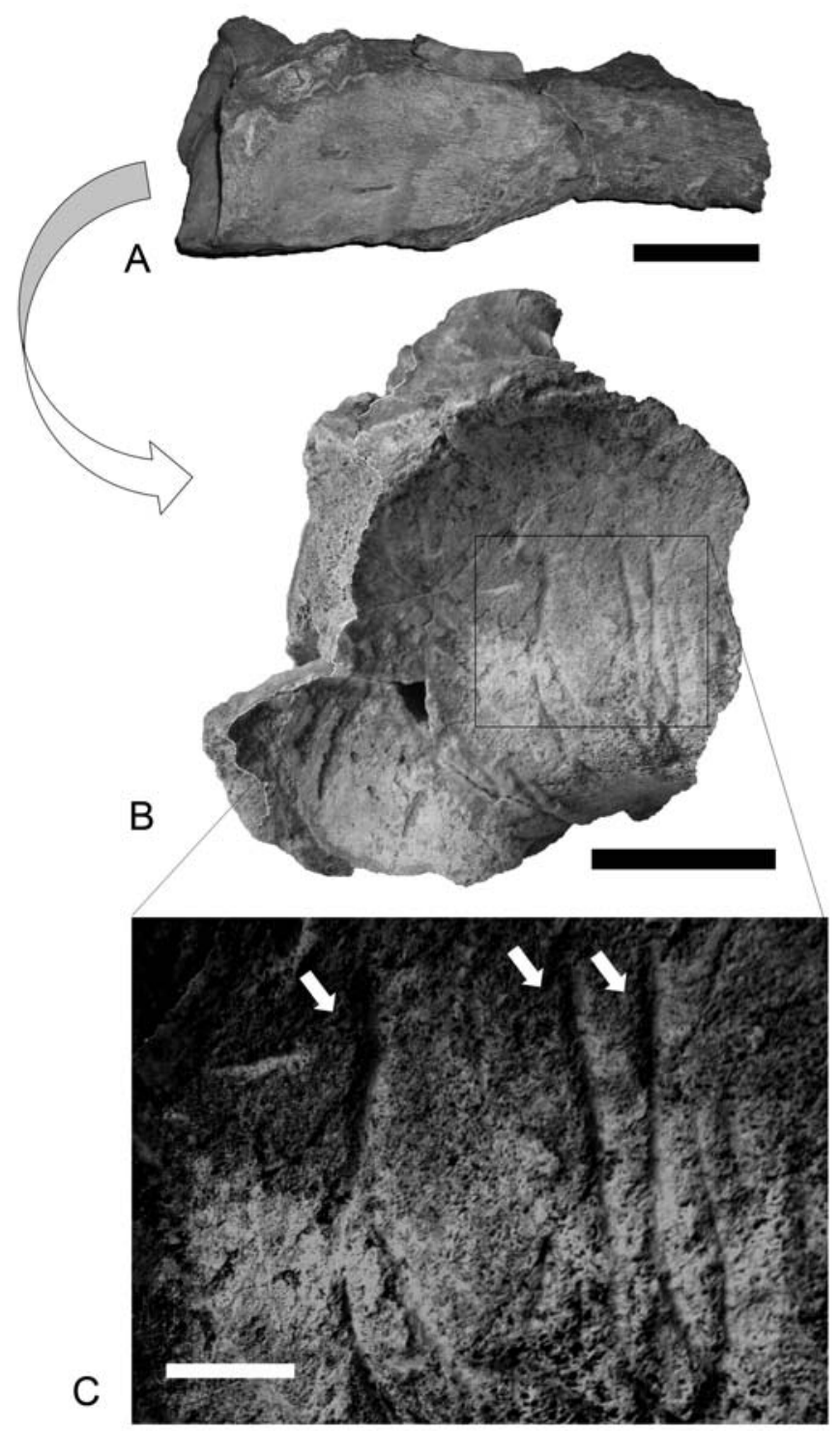

Figura 2. Marcas de dentes em superfície articular proximal de tíbia de Eremotherium laurillardi (MUPHI 1601). A, fragmento de tíbia em vista lateral; $\mathbf{B}$, marcas de dentes na vista proximal da superfície articular da tíbia; $\mathbf{C}$, marcas de dentes indicadas pelas setas. Escalas: $A-B=50 \mathrm{~mm} ; C=10 \mathrm{~mm}$.

Figure 2. Teeth marks in articular proximal surface of tibia of Eremotherium laurillardi (MUPHI 1601). A, tibia fragment in lateral view; $\mathbf{B}$, teeth marks in the proximal view of the articular surface of the tibia; $C$, teeth marks indicated by arrows. Scale bars: $A-B=$ $50 \mathrm{~mm} ; \mathrm{C}=10 \mathrm{~mm}$. instrumentos líticos, no entanto descarta-se a hipótese do homem como gerador dessas feições porque elas apresentam-se paralelas entre si e perpendiculares ao maior eixo do osso. Segundo Fariña \& Castilla (2007) e Bissaro-Júnior (2008), marcas da utilização de ossos por humanos se distribuem aleatoriamente pelo corpo do osso. Além disso, marcas geradas por instrumentos de corte humanos possuem concavidade com formato em "V" (Bunn, 1981; Potts \& Shipman, 1981; Shipman \& Rose, 1983; Bunn \& Kroll, 1986). As marcas aqui observadas apresentam concavidade com formato em "U", o que segundo Haynes (1980b, 1982) e Fiorillo (1988) é característico de mordida de carnívoro.

Nos depósitos quaternários brasileiros, Cerdocyon thous Smith, 1839, Speothos venaticus (Lund, 1842), Dusicyon cf. $D$. avus (Burmeister, 1866), Protocyon troglodytes (Lund, 1842), Crysocyon brachyurus (Illiger, 1815) e Theriodictis Mercerat, 1891, têm sido os táxons de canídeos registrados (Berta, 1984; Gomide, 1989; Guérin et al., 1993; Bergqvist, 1993; Cartelle, 1999; Cartelle \& Langguth, 1999; Lessa et al., 1999; Porpino et al., 2004; Rodrigues et al., 2004; Sedor et al., 2004; Soibelzon \& Prevosti, 2007; Ribeiro \& Scherer, 2009). Além de se restringirem ao sul do país, os registros de Theriodictis sp. e D. avus para o Brasil são duvidosos (Soibelzon \& Prevosti, 2007) e, portanto, não serão discutidos neste trabalho. $C$. thous e $S$. venaticus são animais de pequeno porte, enquanto que os demais têm porte maior. Soibelzon \& Prevosti (2007) consideram C. thous

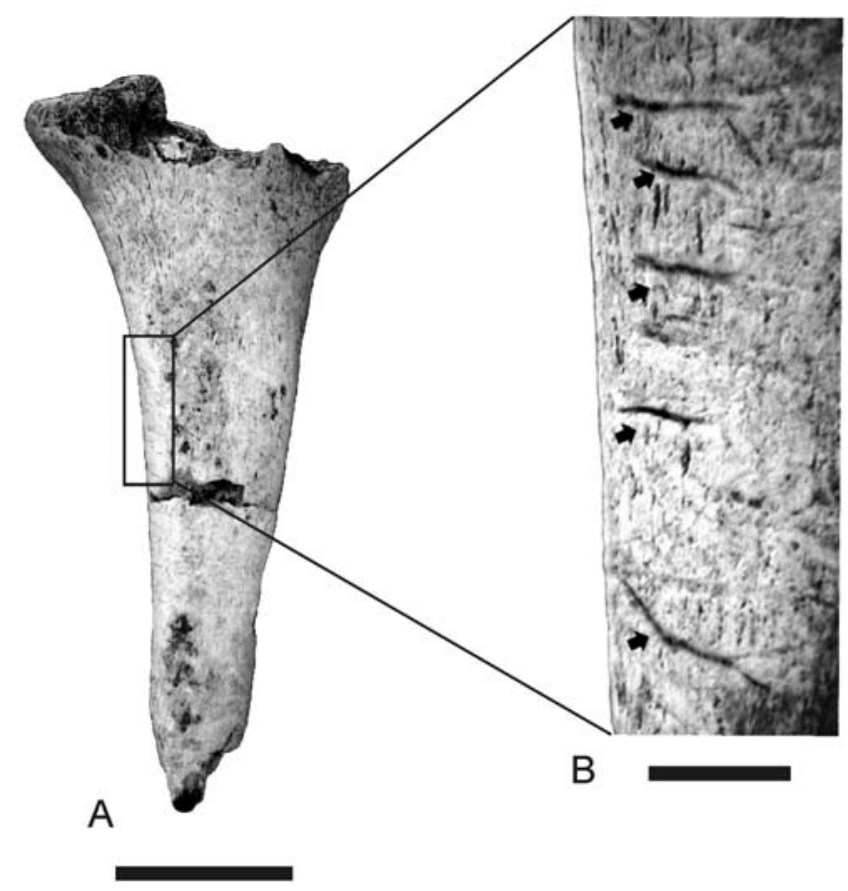

Figura 3. Marcas de dentes posicionadas paralelamente entre si e perpendicularmente em relação ao maior eixo em ulna de Eremotherium laurillardi (MUPHI 1591). A, ulna em vista lateral; B, marcas de dentes indicadas pelas setas. Escalas: $A=100 \mathrm{~mm}$; $B$ $=20 \mathrm{~mm}$.

Figure 3. Teeth marks positioned parallel to each other and perpendicular in relation to the major axis in ulna of Eremotherium laurillardi (MUPHI 1591). A, ulna in lateral view; B, teeth marks indicated by arrows. Scale bars: $A=100 \mathrm{~mm}$; $=20 \mathrm{~mm}$. 
Tabela 1. Ossos de mamíferos do sítio paleontológico de Jirau que apresentam marcas de dentes, e médias das medidas (mm) das marcas para cada elemento esqueletal.

Table 1. Bones of mammals from the paleontological site of Jirau with teeth marks, and mean measurements $(\mathrm{mm})$ of the marks for each skeletal element.

\begin{tabular}{|c|c|c|c|}
\hline Numeração & Elemento esqueletal & $\begin{array}{c}\text { Tamanho das marcas } \\
\text { (comprimento x largura) }\end{array}$ & Táxon com feições \\
\hline MUPHI 1576 & Vértebra & $48,8 \times 3,2$ & Eremotherium laurillardi \\
\hline MUPHI 1577 & Vértebra & $48,3 \times 3,1$ & Eremotherium laurillardi \\
\hline MUPHI 1578 & Vértebra & $50,0 \times 3,0$ & Eremotherium laurillardi \\
\hline MUPHI 1579 & Vértebra & $51,1 \times 3,0$ & Eremotherium laurillardi \\
\hline MUPHI 1580 & Costela & $25,1 \times 3,1$ & Eremotherium laurillardi \\
\hline MUPHI 1581 & Costela & $29,3 \times 3,0$ & Eremotherium laurillardi \\
\hline MUPHI 1582 & Costela & $29,9 \times 3,1$ & Eremotherium laurillardi \\
\hline MUPHI 1583 & Costela & $25,0 \times 3,0$ & Eremotherium laurillardi \\
\hline MUPHI 1584 & Úmero & $50,2 \times 3,0$ & Notiomastodon platensis \\
\hline MUPHI 1585 & Úmero & $50,0 \times 3,1$ & Eremotherium laurillardi \\
\hline MUPHI 1586 & Úmero & $48,9 \times 3,1$ & Eremotherium laurillardi \\
\hline MUPHI 1587 & Úmero & $49,9 \times 3,0$ & Eremotherium laurillardi \\
\hline MUPHI 1588 & Úmero & $50,3 \times 2,7$ & Eremotherium laurillardi \\
\hline MUPHI 1589 & Úmero & $49,9 \times 3,2$ & Eremotherium laurillardi \\
\hline MUPHI 1590 & Úmero & $50,2 \times 3,4$ & Eremotherium laurillardi \\
\hline MUPHI 1591 & Ulna & $27,4 \times 3,0$ & Eremotherium laurillardi \\
\hline MUPHI 1592 & Fêmur & $51,0 \times 3,0$ & Eremotherium laurillardi \\
\hline MUPHI 1593 & Fêmur & $50,0 \times 3,0$ & Eremotherium laurillardi \\
\hline MUPHI 1594 & Tíbia & $48,4 \times 3,1$ & Eremotherium laurillardi \\
\hline MUPHI 1595 & Tíbia & $49,6 \times 3,4$ & Eremotherium laurillardi \\
\hline MUPHI 1596 & Indeterminado & $51,1 \times 2,9$ & - \\
\hline MUPHI 1597 & Astrágalo & $30,0 \times 3,1$ & Glossotherium sp. \\
\hline MUPHI 1598 & Falange & $25,2 \times 3,2$ & Eremotherium laurillardi \\
\hline MUPHI 1599 & Falange & $27,1 \times 3,0$ & Eremotherium laurillardi \\
\hline MUPHI 1600 & Indeterminado & $23,2 \times 2,8$ & - \\
\hline MUPHI 1601 & Tíbia & $52,2 \times 3,4$ & Eremotherium laurillardi \\
\hline
\end{tabular}

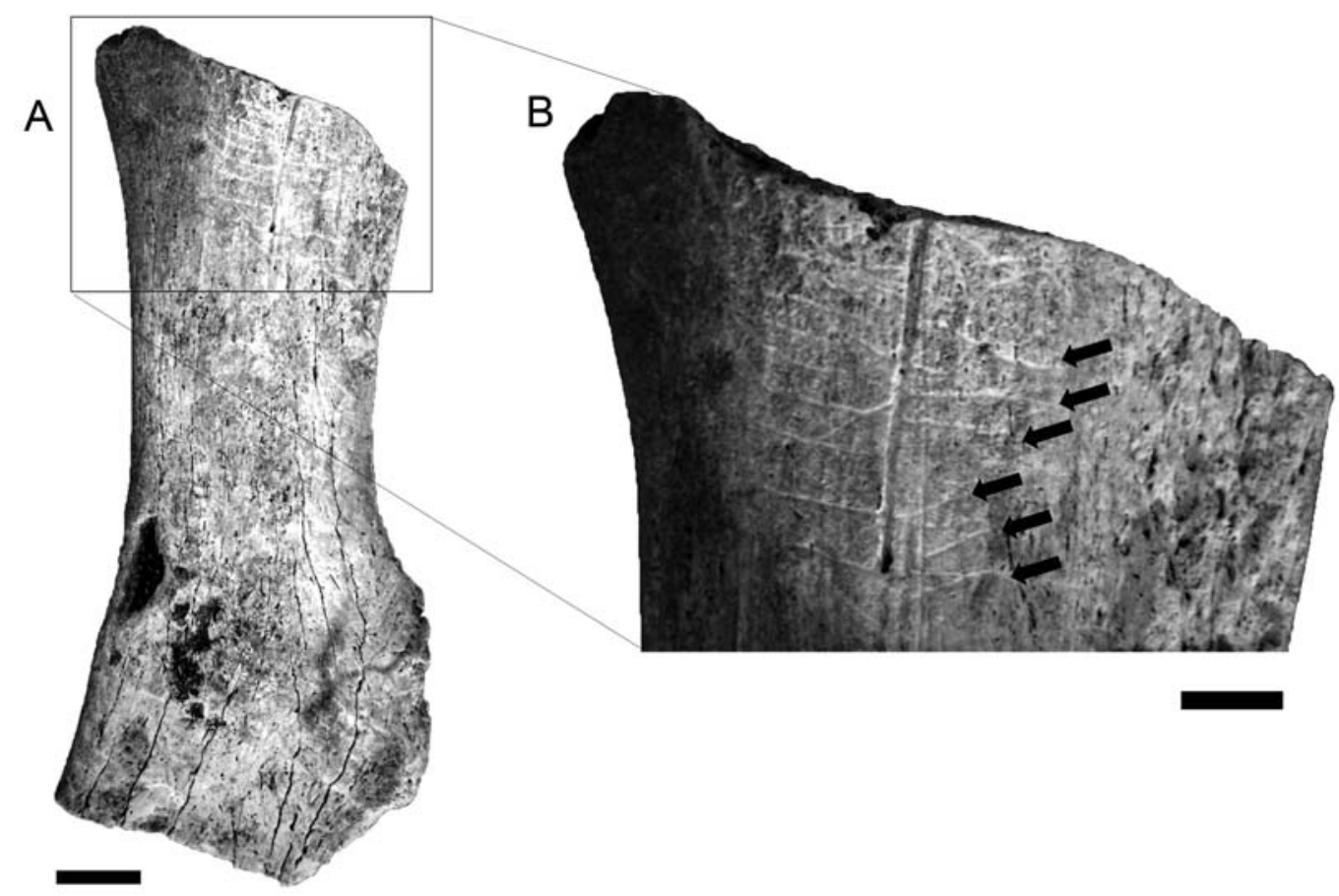

Figura 4. Marcas de dentes em osso longo indeterminado (MUPHI 1596). A, visão geral do osso; B, marcas de dentes perpendiculares em relação ao maior eixo do osso. Escalas: $A=30 \mathrm{~mm} ; B=10 \mathrm{~mm}$.

Figure 4. Teeth marks in indeterminated long bone (MUPHI 1596). A, general view of bone; $\mathbf{B}$, teeth marks perpendicular in relation to the major axis of bone. Scale bars: $A=30 \mathrm{~mm} ; B=10 \mathrm{~mm}$. 
e C. brachyurus como onívoros com dieta baseada principalmente em frutos, roedores, insetos e restos de animais em decomposição. P. troglodytes tem maior abundância de registros nos depósitos pleistocênicos nordestinos (Porpino et al., 2004; Auler et al., 2006), inclusive nos tanques de João Cativo, também em Itapipoca (Gomide, 1989), que distam cerca de $10 \mathrm{~km}$ do Tanque do Jirau. Para Prevosti (2006), Prevosti \& Vizcaíno (2006) e Oliveira et al. (2005) P. troglodytes se alimentaria de mamíferos de médio a grande porte, no entanto, desconsideram a hipótese de que ela predaria megamamíferos. Cartelle (1999), por outro lado, acredita que essa espécie tenha se alimentado apenas de pequenos e médios vertebrados. Paralelamente, Berta (1989) considera as espécies dos gêneros Protocyon como animais carniceiros. Assim sendo, é possível inferir que $P$. troglodytes não predasse megamamíferos pleistocênicos, mas pudesse se alimentar desses animais durante o processo de necrólise.

Dessa forma, considerando-se o porte e a distribuição geográfica, e incluindo a ocorrência em localidade próxima ao sítio estudado, é sugerido Protocyon troglodytes como o candidato natural para a produção das feições estudadas, mesmo este táxon não estando registrado no Tanque do Jirau sob a forma de fóssil corporal até o presente momento. A largura das marcas em todos os espécimes analisados apresentando o mesmo tamanho ( $\pm 3 \mathrm{~mm}$; ver Tabela 1) também sugere que essas feições devam ter sido produzidas por um mesmo predador.

Recentemente, marcas geradas por canídeos (possivelmente Protocyon troglodytes) em elementos esqueletais de depósitos pleistocênicos sul-americanos foram observadas em material atribuído à Notiomastodon platensis (Dominato et al., 2011), sendo desconhecido o registro desse tipo de feição em fósseis de outros mamíferos.

\section{CONSIDERAÇÕES FINAIS}

É registrada pela primeira vez a ocorrência de marcas de dentes de carnívoros/carniceiros em mamíferos pleistocênicos coletados no Nordeste do Brasil. Pelo tamanho das feições produzidas e pela distribuição geográfica e hábitos alimentares dos carnívoros pleistocênicos conhecidos no Brasil, as marcas podem ter sido produzidas por Protocyon troglodytes, mesmo esse táxon não estando registrado no Tanque do Jirau sob a forma de fóssil corporal até o presente momento. Registra-se também, pela primeira vez, a interação alimentar entre Carnivora e Xenarthra no Pleistoceno tardio da América do Sul.

\section{AGRADECIMENTOS}

Os autores agradecem a C.L. Ximenes e A.S.T. dos Santos, curador e diretor do Museu de Pré-história de Itapipoca, respectivamente, por cederem o material do Tanque do Jirau para estudo; G. Haynes pelo auxílio na identificação das marcas de dentes; A.M. Ribeiro pelas valiosas sugestões; B. de Aquino pelo auxílio com as imagens realizadas na Universidade Federal do Rio de Janeiro. À Coordenação de Aperfeiçoamento de Pessoal de Nível Superior (CAPES) e à
Fundação Carlos Chagas Filho de Amparo à Pesquisa do Estado do Rio de Janeiro (FAPERJ) pelo suporte financeiro. Aos dois revisores anônimos pelos comentários que contribuíram para melhoria deste trabalho.

\section{REFERÊNCIAS}

Andrews, P. 1990. Owls, caves and fossils. London, The Natural History Museum, $231 \mathrm{p}$.

Araújo-Júnior, H.I. \& Porpino, K.O. 2011. Assembleias fossilíferas de mamíferos do Quaternário do Estado do Rio Grande do Norte, Nordeste do Brasil: diversidade e aspectos tafonômicos e paleoecológicos. Pesquisas em Geociências, 38(1):67-83.

Auler, A.S.; Piló, L.B.; Smart, P.L.; Wang, X.; Hoffmann, D.; Richards, D.A.; Edwards, R.L.; Neves, W.A. \& Cheng, H. 2006. U-series dating and taphonomy of Quaternary vertebrates from Brazilian caves. Palaeogeography, Palaeoclimatology, Palaeoecology, 240:508-522. doi:10.1016/j.palaeo.2006.03.002

Avilla, L.S.; Dominato, V.H.S.; Pêgo, F.B. \& Silva, R.C. 2009. A tafonomia como ferramenta de reconhecimento de relações ecológicas: um estudo de caso sobre a interação presa-predador a partir de restos fósseis encontrados na Gruta dos Moura, Aurora do Tocantins, Brasil. In: CONGRESSO DE ECOLOGIA DO BRASIL, 9, 2009. Anais, São Lourenço, p. 1-3.

Behrensmeyer, A.K. 1978. Taphonomic and ecologic information from bone weathering. Paleobiology, 4(2):150-162.

Bergqvist, L.P. 1993. Jazimentos pleistocênicos do Estado da Paraíba e seus fósseis. Revista Nordestina de Biologia, 8(2):143-158.

Berta, A. 1984. The Pleistocene bush dog Speothos pacivorus (Canidae) from the Lagoa Santa caves, Brazil. Journal of Mammalogy, 65(4):549-559.

Berta, A. 1989. Quaternary evolution and biogeography of the large South American Canidae (Mammalia: Carnivora). University of California Publication, Geological Sciences, 132: 1-149.

Bissaro-Júnior, M.C. 2008. Tafonomia como ferramenta zooarqueológica de interpretação: viés de representatividade óssea em sítios arqueológicos, paleontológico e etnográfico. Programa de Pós-Graduação em Ecologia de Ecossistemas Terrestres e Aquáticos, Universidade de São Paulo, Dissertação de Mestrado, 102 p.

Britt, B.B.; Eberth, D.A.; Scheetz, R.D.; Greenhalgh, B.W. \& Stadtman, K.L. 2009. Taphonomy of debris-flow dinosaur bonebeds at Dalton Wells, Utah (Lower Cretaceous, Cedar Mountain Formation, USA). Palaeogeography, Palaeoclimatology, Palaeoecology, 280:1-22. doi:10.1016/j.palaeo.2009.06.004

Bunn, H.T. 1981. Archaeological evidence for meat-eating by PlioPleistocene hominids from Koobi Fora and Olduvai Gorge. Nature, 291:574-577.

Bunn, H.T. \& Kroll, E. 1986. Systematic butchery by Plio/ Pleistocene hominids at Olduvai Gorge, Tanzania. Current Anthropology, 27:431-452.

Cartelle, 1998. Um pequeno urso do Pleistoceno Final da Bahia. Acta Geologica Leopoldensia, 21(46/47):171-189.

Cartelle, C. 1999. Pleistocene mammals of the Cerrado and Caatinga of Brazil. In: J.F. Eisenberg \& K.H. Redford (eds.) Mammals of the Neotropics: the Central Neotropics, University of Chicago Press, p. 27-46.

Cartelle, C. \& Langguth, A. 1999. Protocyon troglodytes (Lund): um canídeo intertropical extinto. Anais da Academia Brasileira de Ciências, 71:371-384.

Dominato, V.H.S.; Mothé, D.; Costa-da-Silva, R. \& Avilla, L.S. 
2011. Evidence of scavenging on remains of the gomphothere Haplomastodon waringi (Proboscidea: Mammalia) from the Pleistocene of Brazil: taphonomic and paleoecological remarks. Journal of South American Earth Sciences, 31:271-277. doi:10.1016/j.jsames.2011.01.002

Fariña, R. \& Castilla, R. 2007. Earliest evidence for humanmegafauna interaction in the Americas. In: M.E. Corona \& J. Arroyo-Cabrales (eds.) Human and faunal relationships reviewed: an archaeozoological approach, British Archaeological Reports, International Series, 1627:31-34.

Fiorillo, A.R. 1984. An introduction to the identification of trample marks. Current Research in the Pleistocene, 1:47-48.

Fiorillo, A.R. 1988. Taphonomy of Hazard homestead Quarry (Ogallala Group), Hitchcock County, Nebraska. Contributions to Geology, 26:57-97.

Fiorillo, A.R. 1991. Taphonomy and depositional setting of Careless Creek Quarry (Judith River Formation), Wheatland County, Montana, USA. Palaeogeography, Palaeoclimatology, Palaeoecology, 81:281-311.

Gomide, M. 1989. Mamíferos pleistocênicos de Itapipoca, Ceará, Brasil, depositados no Museu Nacional, Rio de Janeiro. Programa de Pós-Graduação em Zoologia, Universidade Federal do Rio de Janeiro, Dissertação de Mestrado, 173 p.

Gomide, M.; Bergqvist, L.P. \& Rêgo, D.D. 1987. O "tigre-dentede-sabre" (Smilodon populator) de Itapipoca, Ceará. In: CONGRESSO BRASILEIRO DE PALEONTOLOGIA, 10, 1987. Anais, Rio de Janeiro, UFRJ, p. 197-207.

Guérin, C.; Hugueney, M.; Mourer-Chauviré, C. \& Faure, M. 1993. Paléoenvironement Pléistocène dans l'aire archéologique de São Raimundo Nonato (Piaui, Brésil): apport des mammifères et des oiseaux. Documentation du Laboratóire de Géologie de Lyon, 125:187-202.

Haynes, G. 1980a. Evidence of carnivore gnawing on Pleistocene and Recent mammalian bones. Paleobiology, 6(3):341-351.

Haynes, G. 1980b. Prey bones and predators: potencial ecologic information from analyses of bone sites. Ossa, 7:75-97.

Haynes, G. 1982. Utilization and skeletal disturbances of North American prey carcasses. Arctic, 35(2):266-281.

Haynes, G. 1983. A guide for differentiating mammalian carnivore taxa responsible for gnaw damage to herbivore limb bones. Paleobiology, 9(2):164-172.

Kinoshita, A.; Barreto, A.M.F.; Almeida, J.A.C.; Figueiredo, A.M.; Nicolucci, P.; Graeff, C.F.O. \& Baffa, O. 2005. ESR dating at K and $\mathrm{X}$ band of northeastern Brazilian Megafauna. Applied Radiation and Isotopes, 62:225-229.

Kinoshita, A.; Barreto, A.M.F.; Alves, R.S.; Figueiredo, A.M.; Sarkis, J.E.S.; Dias, M.L. \& Baffa, O. 2008. ESR dating of teeth from northeastern Brazilian Megafauna. Radiation Measurements, 43:809-812. doi:10.1016/j.radmeas.2007.11.075

Lessa, G.; Cartelle, C.; Faria, H.D. \& Gonçalves, P.R. 1999. Novos achados de mamíferos carnívoros do Pleistoceno Final-Holoceno em grutas calcárias do Estado da Bahia. Acta Geologica Leopoldensia, 21(46/47):157-169.

Lyman, R.L. 1994. Vertebrate Taphonomy. Cambridge, Cambridge University Press, 524 p.

Mothé, D.; Avilla, L.S.; Cozzuol, M.A. \& Winck, G.R. no prelo. Revision of the Quaternary South America lowland gomphotheres (Mammalia: Proboscidea: Gomphotheriidae). Quaternary International. doi:10.1016/j.quaint.2011.05.018

Oliveira, E.V.; Barreto, A.M.F. \& Alves, R.S. 2009. Aspectos sistemáticos, paleobiogeográficos e paleoclimáticos dos mamíferos quaternários de Fazenda Nova, PE, Nordeste do Brasil. Gaea, 5(2):75-85. doi:10.4013/gaea.2009.52.004

Oliveira, E.V.; Prevosti, F.J. \& Pereira, J.C. 2005. Protocyon troglodytes (Lund) (Mammalia, Carnivora) in the Late Pleistocene of Rio Grande do Sul, and their paleoecological significance. Revista Brasileira de Paleontologia, 8(3):215-220.

Palmqvist, P. \& Arribas, A. 2001. Taphonomic decoding of the paleobiological information locked in a lower Pleistocene assemblage of large mammals. Paleobiology, 27(3):512-530.

Porpino, K.O.; Santos, M.F.C.F. \& Bergqvist, L.P. 2004. Registro de mamíferos fósseis no Lajedo de Soledade, Apodi, Rio Grande do Norte, Brasil. Revista Brasileira de Paleontologia, 7(3):349-358.

Potts, R. \& Shipman, P. 1981. Cutmarks made by stone tools on bones from Olduvai Gorge, Tanzania. Nature, 291:577-580.

Prevosti, F.J. 2006. Grandes canidos (Carnivora, Canidae) del Cuaternario de la Republica Argentina: sistematica, filogenia, bioestratigrafia y paleoecologia. Facultad de Ciencias Naturales y Museo, Universidad Nacional de La Plata, Tese de Doutorado, $506 \mathrm{p}$.

Prevosti, F.J. \& Vizcaíno, S.F. 2006. Paleoecology of the large carnivore guild from the Late Pleistocene of Argentina. Acta Paleontologica Polonica, 51(3):407-422.

Ribeiro, A.M. \& Scherer, C.S. 2009. Mamíferos do Pleistoceno do Rio Grande do Sul, Brasil. In: A.M. Ribeiro, S.G. Bauermann \& C.S. Scherer (eds.) Quaternário do Rio Grande do Sul: integrando conhecimentos, Monografias da Sociedade Brasileira de Paleontologia, 1:171-191.

Rodrigues, P.H.; Prevosti, F.J.; Ferigolo, J. \& Ribeiro, A.M. 2004. Novos materiais de Carnivora para o Pleistoceno do Estado do Rio Grande do Sul, Brasil. Revista Brasileira de Paleontologia, 7(1):77-86.

Sedor, A.F.; Born, P.A. \& Santos, F.M.S. 2004. Fósseis pleistocênicos de Scelidodon (Mylodontidae) e Tapirus (Tapiridae) em cavernas paranaenses (PR, sul do Brasil). Acta Biologica Paranaense, 33(1-4):121-128.

Shipman, P. 1981. Life history of a fossil: an introduction to taphonomy and paleoecology. Cambridge, Harvard University Press, $222 \mathrm{p}$.

Shipman, P. \& Rose, J. 1983. Early hominid hunting, butchering, and carcass-processing behaviors: approaches to the fossil record. Journal of Anthropological Archaeology, 2: 57-98.

Soibelzon, L. \& Prevosti, F.J. 2007. Los carnívoros (Carnivora, Mammalia) terrestres del Cuaternario de América del Sur. In: G.X. Pons \& D. Vicens (eds.) Geomorfologia litoral $i$ Quaternari, Monografías de la Societat D'història Natural de les Balears, p. 49-68.

Trajano, R. \& Ferrarezzi, H. 1994. A fossil bear from northeastern Brazil with a phylogenetic analysis of the South American extinct Tremarctinae (Ursidae). Journal of Vertebrate Paleontology, 14(4):552-561.

Ximenes, C.L.; Santos, A.S.T. \& Monteiro, F.A.C. 2010. Nota preliminar sobre os fósseis de vertebrados do Sítio Paleontológico do Jirau, Pleistoceno de Itapipoca, Estado do Ceará. In: SIMPÓSIO BRASILEIRO DE PALEONTOLOGIA DE VERTEBRADOS, 7, 2010. Boletim Informativo Paleontologia em Destaque, Edição Especial, Rio de Janeiro, p. 122.

Received in March, 2011; accepted in November, 2011. 